\title{
ITO-Free, Compact, Color Liquid Crystal Devices Using Integrated Structural Color Filters and Graphene Electrodes
}

\author{
Jinbao Guo, Chad M. Huard, Yang Yang, Young Jae Shin, Kyu-Tae Lee, and L. Jay Guo*
}

Liquid crystal display (LCD) technology currently dominates the display market, and is by far the largest user of the transparent electrode material indium tin oxide (ITO). ITO is currently the standard transparent electrode used in nearly all electric and optical devices. However, the use of ITO as a transparent electrode has certain limitations, mainly resulting from increased costs due to the increasing scarcity of indium, brittleness, chemical instability, and a non-uniform absorption across the visible spectrum. ${ }^{[1]}$ Recent developments in nanotechnology have opened up an effective strategy to develop new transparent conductive materials, such as graphene, metallic nanostructures, carbon nanotubes and so on. ${ }^{[2-6]}$ Among these options, graphene is a particularly promising material for transparent electrodes in electronic devices due to its high carrier mobility, high transparency and excellent mechanical flexibility, ${ }^{[7-12]}$ Currently methods for fabricating high-quality, large-area graphene by chemical vapor deposition (CVD) have accelerated its practical applications for replacing ITO as transparent electrodes. ${ }^{[13-17]}$

Meanwhile, LCD technologies are faced with a popular trend to get thinner, lighter weight, and more energy efficiency. An effective strategy towards advancing LCD technologies is to integrate multiple functionalities into the each of the components of the LC system and reduce the number of element layers. Among the several possible uses of nanotechnology in LCDs, the development of structural color filters for display applications is very promising. ${ }^{[18,19]}$ For example, besides the essential color filtering effect, the top and bottom metal layers of a metal-dielectric-metal (MDM) Fabry-Perot (F-P) cavity filter can be used as electrodes, replacing one layer of ITO in

Dr. J. B. Guo, C. M. Huard, Y. Yang,

K.-T. Lee, Prof. L. J. Guo

Department of Electrical Engineering

and Computer Science

University of Michigan

Ann, Arbor, Michigan 48109, USA

E-mail: guo@umich.edu

Dr. J. B. Guo

College of Materials Science and Engineering

Beijing University of Chemical Technology

Beijing 100029, PR China

Y. Yang

Tianjin Key Laboratory of Low Dimensional

Materials Physics and Preparing Technology

School of Science, Tianjin University

Tianjin 300072, PR China

Y. J. Shin

Department of Macromolecular Sciences and Engineering

University of Michigan

Ann Arbor, Michigan 48109, USA

DOI: 10.1002/adom.201300525 the LCD structure and leading to compact LC devices with thickness scales that are smaller than those currently used.

In this communication, we demonstrate an ITO-free and more compact color LCD structure. The key to our design is the integration of a structural color filter and a graphene electrode to control the LC orientation, thereby eliminating two layers of ITO from the currently dominant LCD design. Figure 1a presents the schematic diagram of the conventional twistednematic (TN)-LCs device using ITO electrodes and dye-based color filters. Specifically, $90^{\circ}$ TN-LCs devices were fabricated in this study. Note that the principle can also be applied to other LCD modes, such as super TN, in-plane switching, advanced fringe field switching and vertically alignment devices. Figure $1 \mathrm{~b}$ shows the ITO-free hybrid LCs device structure, in which single-layer graphene is used as the back electrode and a MDM F-P cavity color filter replaces both the front ITO electrode and the dye-based color filters. The F-P cavity is capable of filtering white light into individual colors across the entire visible spectrum by choosing the appropriate dielectric layer thickness. Compared with the conventional LCD device, F-P MDM cavity filter used here are much more stable than the traditional colored dyes/pigments used today. ${ }^{[20-26]}$ Meanwhile, this new design significantly improves pass bandwidth and compactness while eliminating two layers of ITO.

Our MDM based color filters have several advantages over the traditional chemical dye-based filter. Current chemical dyebased filters have a relatively low efficiency in utilizing the incident light because each R, G, B filter only passes about $1 / 3$ of the incident light energy. This low efficiency impacts the image brightness and the power consumption of the display. While the traditional chemical dye based color filters operate by absorption of stopband wavelengths, our MDM-based filter is based on an optical resonance effect, where a selected color of light is transmitted and other colors are reflected. It has previously been shown that this reflected light can be recycled within the display structure, thereby increasing the energy efficiency of the display. ${ }^{[18,19,23-26]}$

Here the F-P cavity filter is formed by a Ag/poly(methyl methacrylate) (PMMA, $\mathrm{n} \sim 1.48) / \mathrm{Ag}$ three-layer structure, which is fabricated by a simple thin-film deposition and solution process method. Figure 2a shows the basic structure of the MDM three-layer F-P cavity filter, where the thickness of the bottom and top Ag layers are both $25 \mathrm{~nm}$ and the thickness of PMMA is chosen to be $100 \mathrm{~nm}, 130 \mathrm{~nm}$, and $160 \mathrm{~nm}$ to obtain the transmission of $\mathrm{B}, \mathrm{G}$, and $\mathrm{R}$ colors respectively. A cross-sectional scanning electron microscope (SEM) image of a prepared green color filter is displayed in Figure S1, with the measured thickness of each region shown to be in reasonable agreement with the nominal design value. To demonstrate that the color filtering effect is a result of the F-P cavity resonance, and the 
Standard LC device

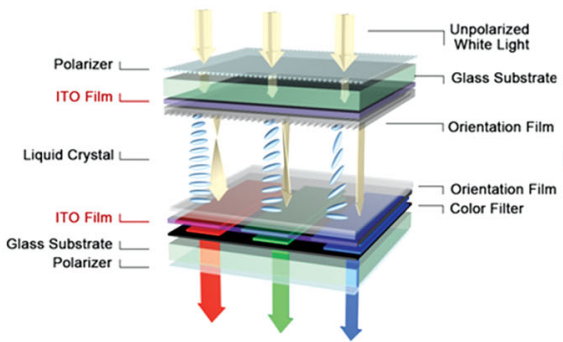

(a)
ITO free LC device

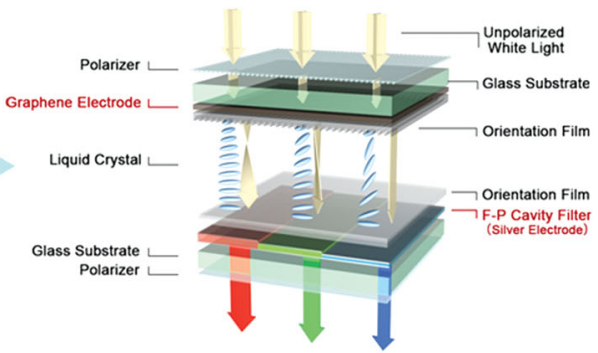

(b)

Figure 1. Schematic illustration of $90^{\circ} \mathrm{TN}-\mathrm{LCs}$ devices based on (a) the conventional ITO electrodes and (b) the graphene back electrode and the Ag front electrode from the F-P cavity filter.
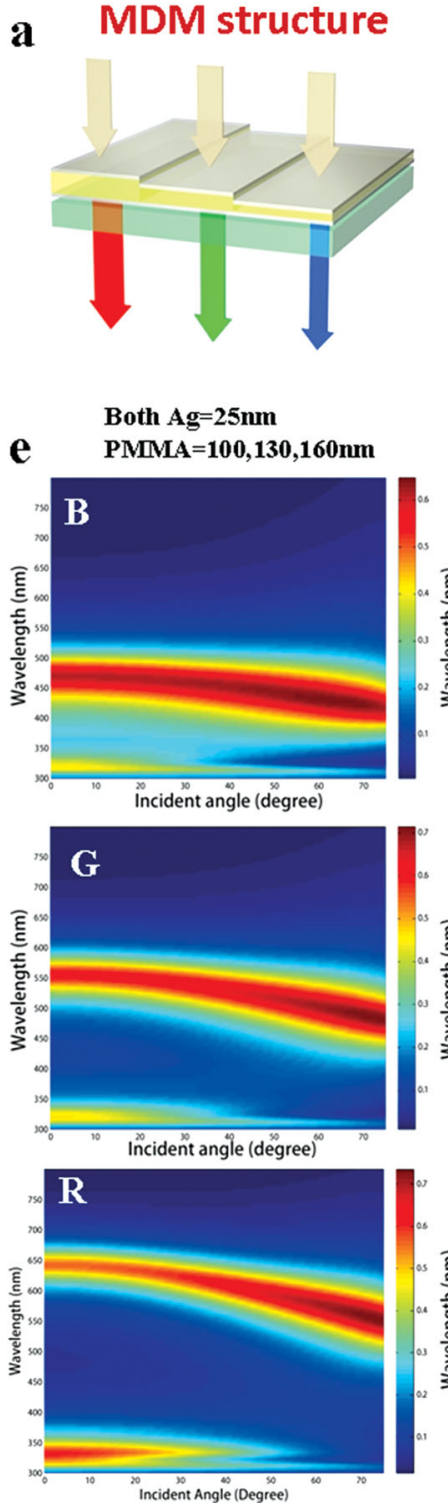

MDMD structure

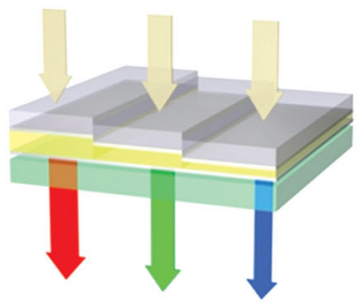

PVA $=80 \mathrm{~nm}$

Both $\mathbf{A g}=\mathbf{2 5 n m}$

PMMA $=100,130,160 \mathrm{~nm}$
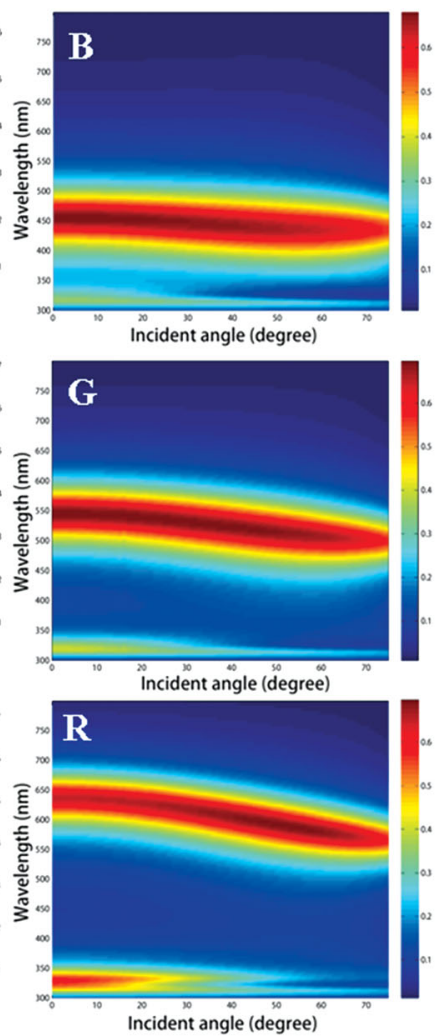

b
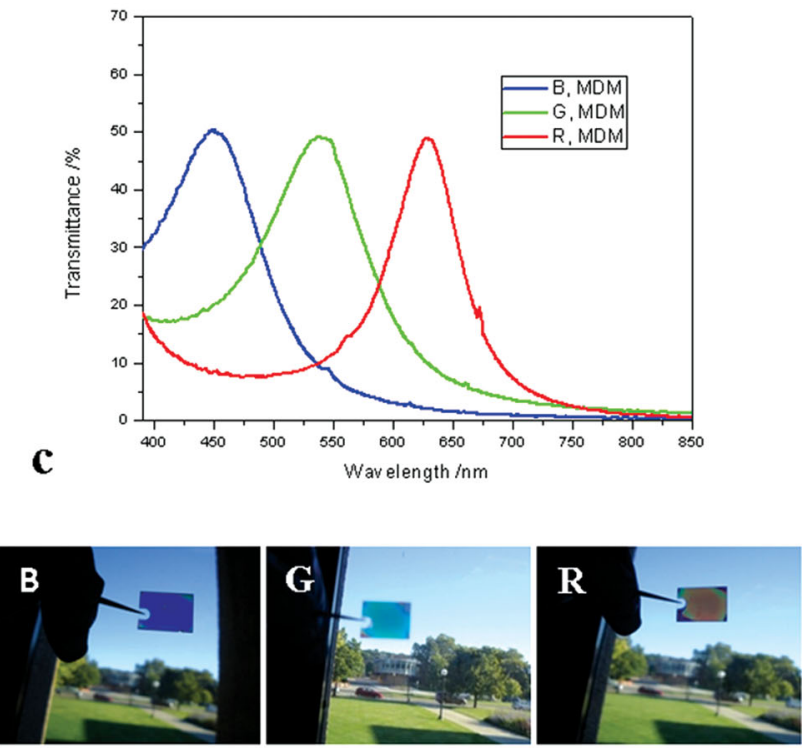

d

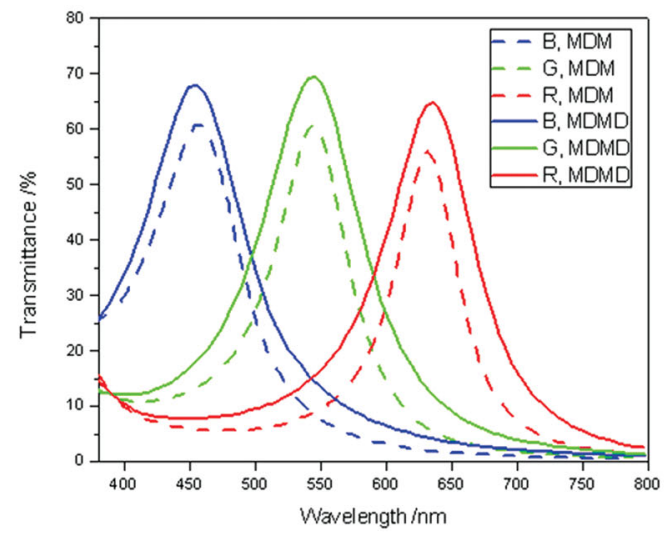

Figure 2. (a) The basic framework of MDM and MDMD F-P cavity filters with RGB colors; (b) measured transmission spectra of MDM with different thickness for the PMMA dielectric layers; (c) the corresponding optical images for RGB color filters; (d) simulated transmission spectra of the MDM and MDMD F-P cavity filters with RGB colors; and (e) simulated angular-dependence of MDM and MDMD F-P cavity filters with RGB colors. 
electric field distribution at the maximum transmission band of the blue color filter is shown in Figure S2. ${ }^{[25,26]}$ Figure 2b shows the measured transmission spectra of three MDM F-P filters with different thickness for the PMMA dielectric layers to produce blue, green and red colors, with measured peak transmission wavelengths of $450 \mathrm{~nm}, 538 \mathrm{~nm}$, and $628 \mathrm{~nm}$ for blue, green and red filters respectively. The peak transmission amplitude of around $50 \%$ can be achieved in all three colors, and the bandwidth of each transmission peak is about $100 \mathrm{~nm}$, which is desirable for LCD applications. Figure 2c shows optical images of each of the RGB color filters.

While our results clearly show that the filtering effect of our devices stems from the optical resonance of the MDM structure, when considering the optical properties of a practical LC device structure, we must also look at the effect of the alignment layer on our color filter. In our device, a polyvinyl alcohol (PVA) layer must be added on top of the MDM color filter to act as an orientation layer for the LCs cell. This additional layer transforms our MDM structure to a MDMD structure as shown in Figure 2a. We used COMSOL multiphysics to simulate the performance of our structure, without and with the PVA layer, to explore the differences between the MDM and MDMD structures. The dielectric constant of Ag is taken from the previous paper and fitted by linear interpolation. ${ }^{[27]}$ From the simulated results, shown in Figure 2d, it can be seen that the PVA layer (the thickness of is $80 \mathrm{~nm}, \mathrm{n} \sim 1.52$ ) serves to significantly increase the transmission intensity. Comparing our measured results to the simulation, we see an excellent match in the main features of the transmission curve, with a slightly lower peak transmission for the fabricated devices. We believe this discrepancy is due to the stronger absorption loss of deposited Ag and possible non-uniformity of the PMMA layer.

In addition to the peak transmission we also used simulation and measurements to investigate the angular- dependence of the optical properties of our color filter, both with and without the alignment layer. Simulation of the angular-dependence of the transmission, shown in Figure 2e, clearly shows that the PVA layer in MDMD structure can help to minimize the angular-dependence of the F-P cavity structure. As a representative example, Figure 3a shows the simulation and experiment results of the wavelength variation $\left(\Delta \lambda_{t} / \lambda_{0}\right)$ with different incident angles for the MDM and MDMD structure of green color filter. We can clearly see from Figure $3 \mathrm{a}$ that the device with a MDMD has the better performance in minimizing angular-dependent resonant wavelength variation. For example, in the MDMD structure, when the incident angle approaches $70^{\circ}$ the variation of the resonant wavelength $\left(\Delta \lambda_{\mathrm{t}} / \lambda_{0}\right)$ is less than $8.5 \%$ (for $\lambda_{\mathrm{t}}=545 \mathrm{~nm}$ at normal incidence, the variation $\Delta \lambda_{\mathrm{t}}$ at $70^{\circ}$ is $\sim 45 \mathrm{~nm}$ ). Meanwhile, the variation $\Delta \lambda_{\mathrm{t}}$ of the MDM structure filter at $70^{\circ}$ is significantly larger at $60 \mathrm{~nm}\left(\Delta \lambda_{\mathrm{t}} / \lambda_{0} \approx 11 \%\right)$. To confirm the simulated results, transmission spectra were measured from the fabricated samples with angles of $20^{\circ}, 40^{\circ}$, and $60^{\circ}$. These measurements were taken using a conventional fiber optic spectrometer with an accurate control of the incident angle for the samples. The colored dots in Figure 3a represent the measured $\Delta \lambda_{\mathrm{t}}$, with each measurement agreeing well with the simulation results for the MDM and MDMD structure of the green color filters.

To gain a better understanding of how each design parameter affects the angular-dependence of our filter performance, we can use a standard F-P cavity theory to model the device analytically. As shown in Figure $3 \mathrm{~b}$, the cavity length is $d$, the refractive index of the dielectric layer is $n$ and the incident angle from air and refraction angle into dielectric layer is $\theta_{1}$ and $\theta_{2}$, respectively. To simplify the model, the absorption and the material dispersion in the system are neglected, but they do not affect the conclusions. Based on the optics relation, the transmittance $T_{\mathrm{FP}}$ of the $\mathrm{F}-\mathrm{P}$ cavity filter can be approximated as: ${ }^{[28]}$

$$
T_{F P}=\frac{(1-R)^{2}}{1+R^{2}-2 R \cos \delta}
$$
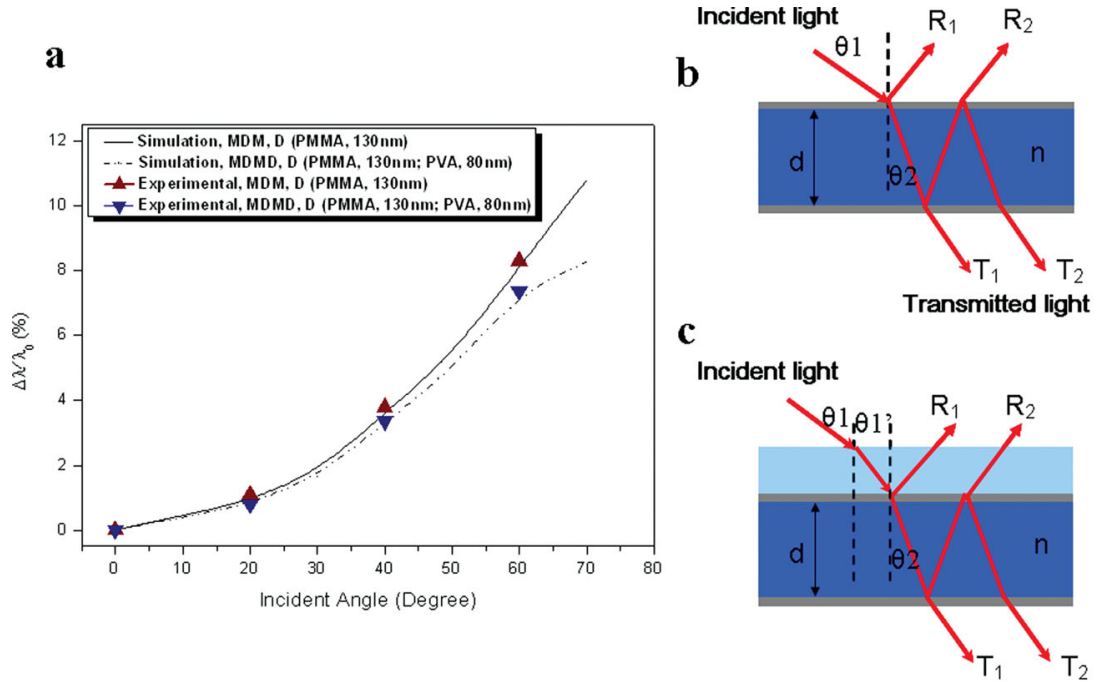

Transmitted light

Figure 3. (a) Simulation (curve) and experiment (color dot) results of MDM and MDMD structures for green color filters on the resonant wavelength variation as a function of incident angles. Here PMMA $(n \sim 1.48)$ and PVA $(n \sim 1.52)$ as materials for the dielectric cavity and top dielectric layer, respectively. $(b, c)$ Schematic of the F-P cavity structure without and with the top dielectric layer, respectively. The incident and refractive angle is $\theta_{1}$ and $\theta_{2}$, respectively. where $R$ is the reflection coefficient of the Ag film, the phase shift $(\delta)$ is the shift experienced between the directly transmitted light $\left(\mathrm{T}_{1}\right)$ and the light which is transmitted after one roundtrip in the cavity $\left(\mathrm{T}_{2}\right)$, which is given by:

$\delta=\frac{4 \pi \cdot n \cdot d}{\lambda} \cdot \cos \theta_{2}$

From Equations (1) and (2), the transmission maximum occurs at the resonant wavelength $\lambda_{\mathrm{t}}$ when the phase difference $\delta=2 m \pi$. Here we only consider the case of $m=1$ because the rest of the resonance modes are sufficiently separated from it to be located in the near infrared band. According to Snell's law, $\sin \theta_{1}=n \cdot \sin \theta_{2}$, so we can get the resonance condition as $\lambda_{t}=2 d \sqrt{n^{2}-\sin ^{2} \theta_{1}}$. Then 
the variation of the resonant wavelength with incident angle $\theta_{1}$ can be calculated as

$$
\left|\Delta \lambda_{r} / \Delta \theta_{1}\right| \approx \frac{2 d \cdot \sin \theta_{1} \cdot \cos \theta_{1}}{\sqrt{n^{2}-\sin ^{2} \theta_{1}}}=\frac{d \cdot \sin 2 \theta_{1}}{\sqrt{n^{2}-\sin ^{2} \theta_{1}}}
$$

Equation (3) shows that the variation of the resonant wavelength is almost inversely proportional to the refractive index of the dielectric layer from Equation (3). This indicates that a highindex dielectric cavity layer (such as titanium dioxide) can lead to color filters with a smaller angular-dependence. In this proofof-principle experiment, we used the PMMA due to the facile solution-process fabrication. In addition, it should be noted that, when using a polymer dielectric, the color filter array (pixel) for RGB colors can be fabricated by using nanoimprinting technology, where PMMA layer is imprinted with a step-like mold of three different depths, forming the RGB colors. On the other hand, as shown in Figure 3c, adding a dielectric layer with a refractive index larger than air reduces the incident angle to the MDM cavity, which can further improve the angle-insensitive behavior according to Equation (3). This result confirms our simulation, and gives an intuitive reason why the PVA layer can improve the angle-independence of the MDM structure.

To compliment our ITO-free front electrode and color filter, we chose to use graphene for the back electrode due to its high transparancy/resistivity ratio, abundant raw materials and ease of scaling. ${ }^{[7,10,11]}$ Previously an electric field-induced Fréedericksz transition was observed in an optical microscope when N-LCs were sandwiched either between a small graphene sheet and a glass substrate coated with ITO. ${ }^{[29]}$ After that, graphene layers from the thermal reduction of graphene oxide film were demonstrated to drive N-LC. ${ }^{[30,31]}$ However, although graphene is expected to become a promising transparent electrode for LCDs, the performance of LC devices based on graphene elec- trodes still face many challenges. Among these challenges, large area, high quality and uniformity of the graphene film each play important limiting roles for display application. As mentioned above, CVD techniques can fabricate layer-controlled graphene sheets over large areas using metal catalyst substrates. ${ }^{[13-17]}$ In this study, graphene fabrication process includes CVD synthesis, coating of graphene with PMMA, copper etching, transferring, drying, and removing of the polymer layer, which is very similar to that of the previous report.[17] The fabrication process was optimized to achieve high-quality, single layer graphene. Graphene was grown on $99.999 \%$ pure copper foil at $1000{ }^{\circ} \mathrm{C}$ for $10 \mathrm{~min}$, with growth pressure of 100 Torr, $\mathrm{CH}_{4}$ flow rate of $10 \mathrm{sccm}$. A detailed fabrication process is described in the experimental section. Figure 4a shows photographic image of a single-layer graphene film $(1.5 \mathrm{~cm} \times 1.5 \mathrm{~cm})$ transferred onto a fused silica $\left(\mathrm{SiO}_{2}\right)$ substrate. As shown in Figure $4 \mathrm{~b}$, a typical optical microscope image of the transferred single-layer film shows almost no color or optical density variation except for the edge region, in which the graphene and $\mathrm{SiO}_{2}$ regions are labeled.

To determine the quality of the graphene film we conducted Raman spectroscopy, morphological studies and electrical measurements. Morphological images of the CVD graphene were taken using SEM and atomic force microscopy (AFM). Both the SEM (Figure 4c) and AFM (Figure 4d) show uniform and clean graphene sheet, with some wrinkles that is one of typical characteristics of the CVD growth and polymer assisted transfer process. ${ }^{[17]}$ Raman spectroscopy measurements confirms that our graphene is single layer, and has a relatively low defect density. Figure 4e shows the Raman spectra of the graphene used in this study, including two characteristic peaks, the $\mathrm{G}$ band at $1589 \mathrm{~cm}^{-1}$ and the 2D band $\left(\sim 2644 \mathrm{~cm}^{-1}\right) \cdot{ }^{[32]}$ The defect-related D-band of the single-layer graphene $\left(1324 \mathrm{~cm}^{-1}\right)$, which is caused by the breathing mode of $\mathrm{sp}^{2}$ atoms and is activated by the existence of defects, is small in our samples.
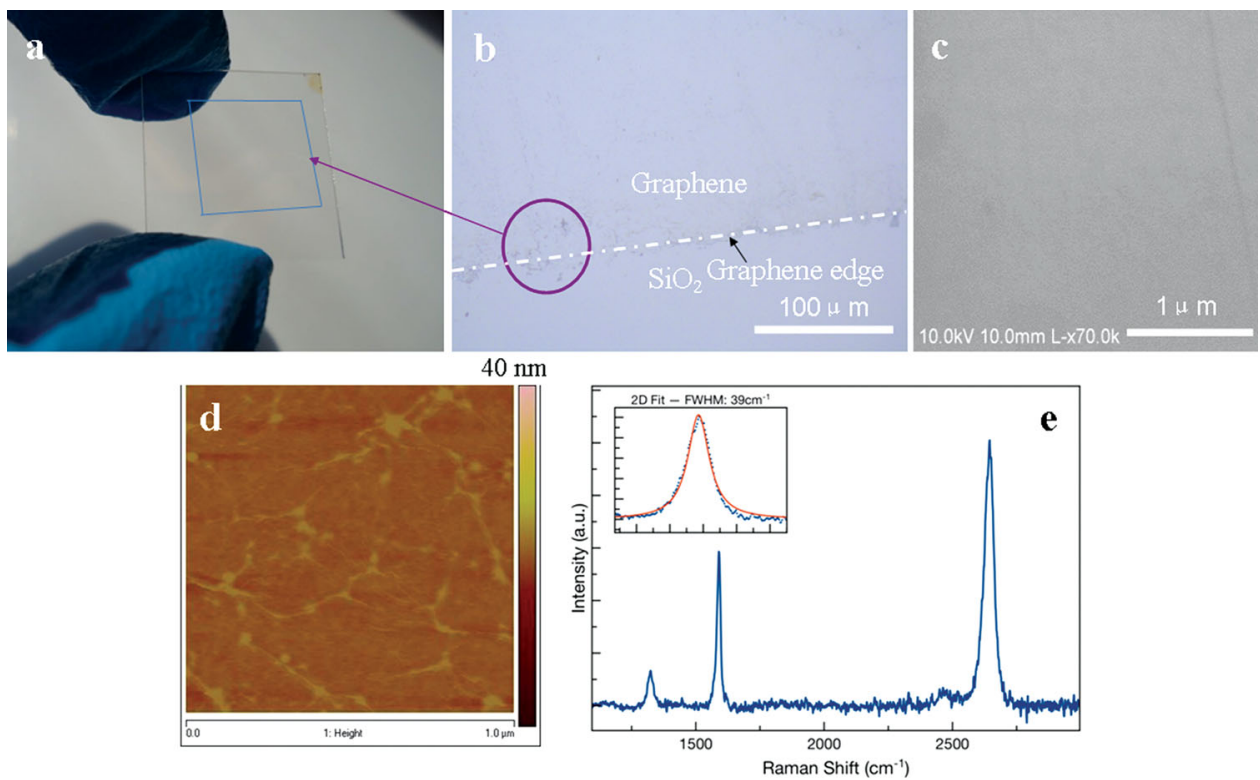

Figure 4. (a) Photographic image of a single-layer graphene film; (b) optical microscope image of single-layer graphene film; (c,d) SEM and AFM images of the single-layer graphene film, and; (e) Raman spectra of the single-layer graphene film. 
a
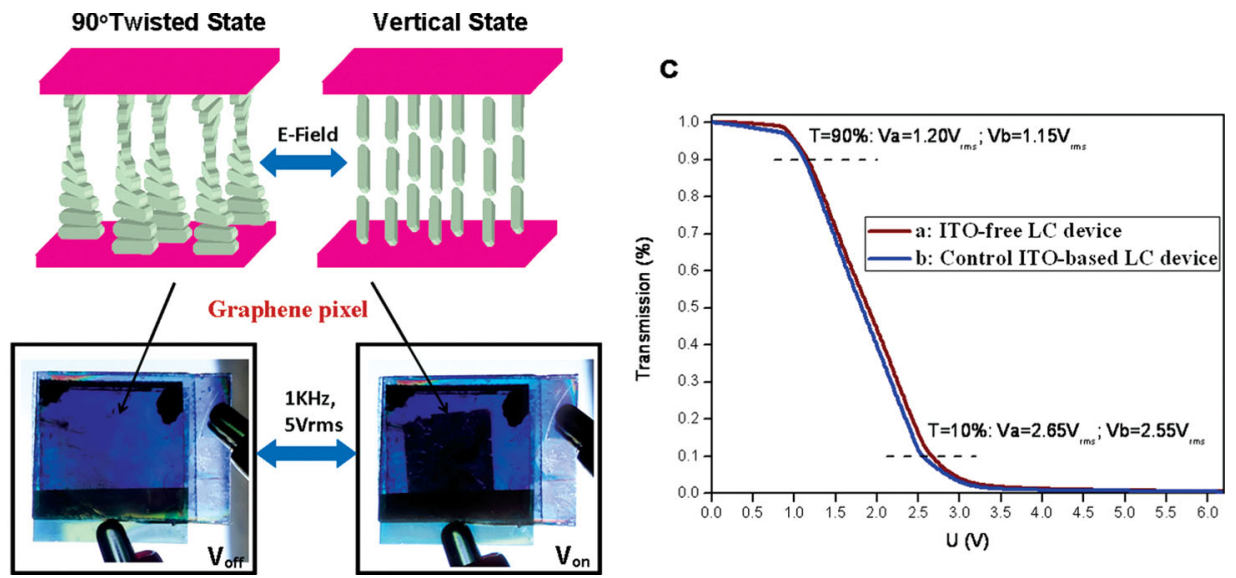

Figure 5. (a) Switching characteristic of $90^{\circ}$ TN-LCs device; (b) photographic images of the LC device between the 'blue' deactivated state without voltage (left) and the 'black' activated state when applied for $5 \mathrm{~V}_{\text {rms }}$ (right), and; (c) transmittance intensity of the LC devices (a: ITO-free LC device and b: Control ITO-based LC device) as a function of the driving voltage.

Additionally, a symmetric 2D band centered at $2644 \mathrm{~cm}^{-1}$ has a full width at half maximum (FWHM) of $39 \mathrm{~cm}^{-1}$, and the corresponding intensity ratio of $I_{2 \mathrm{D}} / I_{\mathrm{G}}$ is approximately 1.73 as shown in the inset image of Figure 4e. All of these Raman spectroscopy results suggest that the high-quality single-layer graphene was achieved as reported in the previous literatures. ${ }^{[17,33]}$ The sheet resistance of our graphene samples was measured to be between 800-1200 $\Omega$ /square using both four-point van der Pauw and transmission line techniques, which is similar with that of the previous reports. ${ }^{[14]}$

In order to evaluate the effectiveness of the MDM electrode/ filter and graphene back electrode we fabricated complete LC device structures as shown schematically in Figure 1b. To fabricate the structure, we first fabricated the graphene electrode and F-P cavity filter on glass substrates. Contacts $(5 / 50 \mathrm{~nm} \mathrm{Ti} /$ $\mathrm{Au}$ ) were deposited one edge of the graphene film to facilitate connection to test equipment. The graphene-electrode and F-P cavity filter were then coated with a thin PVA $(80 \mathrm{~nm})$, and physically rubbed in perpendicular directions to act as an orientation layer and induce a $90^{\circ}$ twisted TN cell. The two glass substrates were seperated by $6 \mu \mathrm{m}$ using polystyrene bead spacers, and capillary-filled with N-LC material. The LC material used in this study was Slichem SLC-1717 LC, which has a birefringence $(\Delta \mathrm{n}=\mathrm{ne}-\mathrm{no})$ of 0.201 . The final device, as tested, can be seen in Figure $5 \mathrm{~b}$. For comparison, a control TN-LC device (ITO-based LC device), consisting of a back ITO electrode and front F-P cavity filter on two glass substrates, was also prepared (Figure S3). Both of these two TN-mode LC devices were fabricated in normally white mode, where the outer surfaces of the glass substrates are laminated with polarizers (supplied by American Polarizers, Inc.) having their transmission direction parallel to the respective rubbing direction of the PVA film on the corresponding glass substrate.

By comparing the measured switching characteristics of our ITO-free LC device with the control LC device, we find that there is no degradation in performance, demonstrating the potential of our ITO-free design. In the initial state, without applied voltage, the blue color of the ITO-free device can be clearly seen in Figure $5 \mathrm{~b}$ (left). When a voltage of $5.0 \mathrm{~V}_{\text {rms }}$ was applied the LC molecules are aligned parallel to the electric field, as shown schematically in Figure 5a, the pixel on the graphene electrode region transform to the black state uniformly, while the other areas still kept blue color state as shown in Figure 5b (right). What's more, the LC cell can be reversibly switched between a blue color state and a black state in the graphene electrode area by controlling the applied voltage, as demonstrated in a video file (see Supporting Information Move1). The intensity change of the LC device at the maximum transmittance band (448 nm) was measured by using a spectrometer as a function of applied voltage. Figure $5 \mathrm{c}$ shows transmitted intensity versus applied voltage ( $\mathrm{f}=1 \mathrm{kHz}$ ) for the ITO-free LC device and the control ITO-based LC device. The threshold voltage (defined as the voltage at which a transmittance of $90 \%$ is obtained) of the ITO-free LC device is measured at $1.20 \mathrm{~V}_{\mathrm{rms}}$, and the transmittance is saturated at $2.65 \mathrm{~V}_{\mathrm{rms}}$ (defined as the voltage at which a transmittance of $10 \%$ is obtained). Comparing this result to the electric-optical curve of the control ITO-based LC device as shown in Figure $5 c$ and Figure $S 3$ (threshold voltage is $1.15 \mathrm{~V}_{\text {rms }}$ and the saturated voltage is $2.55 \mathrm{~V}_{\mathrm{rms}}$ ), we can see there is no increase of the switching voltages in the graphene-based LC device.

Our experimental results demonstrate that, despite our graphene's higher sheet resistance than ITO, there is no observable degradation in performance in this ITO-free device under our experimental conditions. This result may have several explanations. First, considering that the electro-optic switching performance of the device is determined mainly by the proper alignment of LC and the LC ability to respond to a potential difference between the top and bottom electrodes, we expect that the difference in sheet-resistance will have small impact on switching performance at frequencies that are of interest for display technology. ${ }^{[34]}$ For future active matrix LCD displays, as long as the graphene electrode size is small (determined by the pixel size) and controlled by an active transistor, the switching speed of the pixel should not be limited by the sheet resistance of the graphene layer. Second, any defects in the graphene (tears, pinholes, polymer residue) are expected to be small compared to the thickness of the alignment layer, and negligible compared to the thickness of the LC layer. From this perspective we think that any non-uniformity of the LC alignment will be 
minimal, and may be minimized further by alignment to the fringing fields at the defects. Finally, we note that if these issues do become significant in more practical devices or operating conditions, the quality of our graphene electrode is limited by our prototyping technology, and does not represent the peak possible performance of graphene electrodes. Defect density could be reduced by newly developed transfer techniques, and sheet resistance could be improved by doping or integration of metal nanostructures. ${ }^{[35-39]}$ It should also be noted that though this work uses a simple one-pixel ITO-free LC device to show the switching effect, it is not difficult to achieve multi-pixel LC device by patterning the back graphene electrode or the front F-P cavity filter. Active matrix LC devices are also possible by combining the graphene electrode with a transistor (possibly also graphene based) array for practical LCD applications.

In summary, we demonstrated a compact, ITO-free LC device by using a dual-function MDM F-P cavity both as a color filter and an electrode, together with a graphene back electrode to control LC switching. The transmission intensity and the angular-dependence of the F-P filters were measured and analyzed. The fabricated compact color LC device exhibits similar switching performance compared to the control TN-LC device by using an ITO electrode, but with a more compact and economical design. This work shows the potential to use the multifunctional nanostructures for future ITO-free LCs applications.

\section{Experimental Section}

Fabrication of MDM F-P Cavity Filter: First a layer of $\mathrm{Ag}$ with a thickness of $25 \mathrm{~nm}$ was coated glass substrate using physical vapor deposition (PVD) sputtering (Lab 18-2) method. Next a PMMA film of $100 \mathrm{~nm}, 130 \mathrm{~nm}$, and $160 \mathrm{~nm}$ thickness was subsequently made by spin coating method, in which the PMMA 950A2 solution (Microchem, the coating rate: $1600 \mathrm{rpm}$ ) was used to obtain $100 \mathrm{~nm}$ dielectric layer and PMMA 950A3 solution (the coating rate: 4250 and $2750 \mathrm{rpm}$ ) was utilized to achieve dielectric layers with $130 \mathrm{~nm}$ and $160 \mathrm{~nm}$, respectively. Finally, a $\mathrm{Ag}$ film of the $25 \mathrm{~nm}$ thickness as the top metal layer was deposited, again by PVD. The layer thickness of every layer was determined by Dektak 6 M Surface Profilometer.

Preparation of Graphene onto the Glass Substrates: $25 \mathrm{~mm}$ thick copper foil (99.999\%, Alfa Aesar Puratronic) was loaded into an inner quartz tube inside a 2 inch horizontal tube quartz lamp heated, rapid CVD system (Angstrom Engineering). The system was purged with argon gas and evacuated to a vacuum $<10$ mTorr. The sample was then heated to $1000{ }^{\circ} \mathrm{C}$ in $\mathrm{H}_{2}(25 \mathrm{sccm})$ and $\operatorname{Ar}(500 \mathrm{sccm})$ environment with a vacuum level of 100 Torr. The sample is annealed for $30 \mathrm{~min}$ at $1000{ }^{\circ} \mathrm{C}$, then $10 \mathrm{sccm}$ of $\mathrm{CH}_{4}$ is flowed for 10 minutes with $\mathrm{H}_{2}(2 \mathrm{sccm})$ and $\mathrm{Ar}$ (500 sccm). The sample is then cooled rapidly to room temperature. After the CVD synthesis, one side of the copper sample with single-layer graphene is coated with 950PMMA A9 (Microchem) resist and cured at room temperature for $1 \mathrm{~h}$. The other side of the sample is exposed to $\mathrm{O}_{2}$ plasma for $30 \mathrm{~s}$ to remove the graphene on that side. The sample is then left in ferric chloride copper etchant (DalPro) solution for at least $1 \mathrm{~h}$ to completely dissolve away the copper layer. The sample is transferred onto a glass substrate. The PMMA coating is removed with acetone and the substrate is rinsed with deionized water several times.

Fabrication of TN-LC Cell: In this study, SLC-1717 $\left(20^{\circ} \mathrm{C}, 589 \mathrm{~nm}, \Delta \mathrm{n}\right.$ $=0.201$; Slichem LC Material of China) was used as the bulk N-LCs. In order to induce orientation of LC molecules, the inner surfaces of Ag/ PMMA/Ag and graphene layer were coated with a $3.0 \mathrm{wt} \%$ PVA aqueous solution, respectively. The deposited film was pre-baked at $90.0^{\circ} \mathrm{C}$ for $1.0 \mathrm{~min}$ and then oven baked at $120^{\circ} \mathrm{C}$ for about $1.5 \mathrm{~h}$. The PVA layer was subsequently rubbed with a textile cloth under a pressure of $2.0 \mathrm{~g} /$ $\mathrm{cm}^{2}$ along one direction. Next, the two glass substrates were kept apart using spacer of $6 \mu \mathrm{m}$ PS beads as spacers, and were then bonded together with PVA glue, making sure the rubbing directions of the top and bottom layer on the glass substrate are perpendicular to induce $90^{\circ}$ rotation of the LC.

Measurements: SEM imaging was performed using a JEOL JSM-840 SEM; AFM measure was carried out by using NanoMan AFM. Optical microscope images were obtained by Nikon Eclipse Lv150 microscope. Transmission was measured using an HR4000CG spectrometer (Ocean Optics, Inc., Dunedin, FL, USA) and a AvaLight-HAL light, (also Ocean Optics); The electric field is applied to the cells using a function generator (AFG3101, Tektronix). The sheet resistance was measured by four point probe method (Miller FPP-5000 4-Point Probe).

\section{Supporting Information}

Supporting Information is available from the Wiley Online Library or from the author.

\section{Acknowledgements}

J. B. Guo and C. M. Huard contributed equally to this study. This work was supported in part by the National Science Foundation (ECCS1202046 and DMR-1120923). J. B. Guo acknowledges the financial support from National Natural Science Foundation (Grant No. 51373013) and partial support of China Scholarship Council (CSC).

Received: December 18, 2013 Revised: January 28, 2014 Published online: March 7, 2014

[1] D. S. Hecht, L. B. Hu, G. Irvin, Adv. Mater. 2011, 23, 1482-1513.

[2] J. Y. Lee, S. T. Connor, Y. Cui, P. Peumans, Nano. Lett. 2008, 8 , 689-692.

[3] S. De, T. M. Higgins, P. E. Lyons, E. M. Doherty, P. N. Nirmalraj, W. J. Blau, J. J. Boland, J. N. Coleman, ACS Nano 2009, 3, 1767-1774

[4] M. G. Kang, T. Xu, H. J. Park, X. G. Luo, L. J. Guo, Adv. Mater. 2010 22, 4378-4383.

[5] S. I. Na, S. S. Kim, J. Jo, D. Y. Kim, Adv. Mater. 2008, 20, 4061-4067.

[6] W. Gaynor, G. F. Burkhard, M. D. McGehee, P. Peumans, Adv. Mater. 2011, 23, 2905-2910.

[7] S. P. Pang, Y. Hernandez, X. L. Feng, K. Müllen, Adv. Mater. 2011, 23, 2779-2795

[8] S. K. Bae, S. J. Kim, D. Shin, J.-H. Ahn, B. H. Hong, Phys. Scr. 2012, T146, 014024.

[9] F. Bonaccorso, Z. Sun, T. Hasan, A. C. Ferrari, Nat. Photonics 2010 , $4,611-622$

[10] N. O. Weiss, H. L. Zhou, L. Liao, Y. Liu, S. Jiang, Y. Huang, X. F. Duan, Adv. Mater. 2012, 24, 5782-5825.

[11] X. Huang, Z. Y. Zeng, Z. X. Fan, J. Q. Liu, H. Zhang, Adv. Mater. 2012, 24, 5979-6004.

[12] K. S. Novoselov, V. I. Fal'ko, L. Colombo, P. R. Gellert, M. G. Schwab, K. Kim, Nature 2012, 490, 192-200.

[13] J. Coraux, A. T. N'Diaye, C. Busse, T. Michely, Nano. Lett. 2008, 8, $565-570$.

[14] K. S. Kim, Y. Zhao, H. Jang, S. Y. Lee, J. M. Kim, K. S. Kim, J. H. Ahn, P. Kim, J. Y. Choi, B. H. Hong, Nature 2009, 457, 706-710.

[15] Y. Lee, S. Bae, H. Jang, S. Jang, S. E. Zhu, S. H. Sim, Y. I. Song, B. H. Hong, J. H. Ahn, Nano. Lett. 2010, 10, 490-493. 
[16] A. Reina, X. Jia, J. Ho, D. Nezich, H. Son, V. Bulovic, M. S. Dresselhaus, J. Kong, Nano. Lett. 2008, 9, 30-35.

[17] X. Li, W. Cai, J. An, S. Kim, J. Nah, D. Yang, R. Piner, A. Velamakanni, I. Jung, E. Tutuc, S. K. Banerjee, L. Colombo, R. S. Ruoff, Science 2009, 324, 1312-1314.

[18] T. Xu, Y. K. Wu, X. G. Luo, L. J. Guo, Nat. Commun. 2010, 1, 59-62.

[19] T. Xu, H. F. Shi, Y. K. Wu, A. F. Kaplan, J. G. Ok, L. J. Guo, Small 2011, 7, 3128-3136.

[20] C. Genet, T. W. Ebbesen, Nature 2007, 445, 39-46.

[21] K. Kumar, H. G. Duan, R. S. Hegde, S. C. W. Koh, J. N. Wei, J. K. W. Yang, Nat. Nanotechnol. 2012, 7, 557-561.

[22] Y. K. R. Wu, A. E. Hollowell, C. Zhang, L. J. Guo, Scientific Reports 2013, 3, 1194

[23] A. F. Kaplan, T. Xu, L. J. Guo, Appl. Phys. Lett. 2011, 99, 143111.

[24] E. Laux, C. Genet, T. Skauli, T. W. Ebbesen, Nat. Photonics 2008, 2, 161-164.

[25] K. Diest, J. A. Dionne, M. Spain, H. A. Atwater, Nano. Lett. 2009, 9, 2579-2583.

[26] Y. T. Yoon, S. S. Lee, Optics Express 2010, 18, 5344-5349.

[27] E. D. Palik, Handbook of Optical Constants of Solids, Academic Press, Boston 1985.

[28] M. Born, E. Wolf, Principles of Optics, 7th ed., Cambridge University Press, New York 1999.

[29] P. Blake, P. D. Brimicombe, R. R. Nair, T. J. Booth, D. Jiang, F. Schedin, L. A. Ponomarenko, S. V. Morozov, H. F. Gleeson,
E. W. Hill, A. K. Geim, K. S. Novoselov, Nano. Lett. 2008, 8, 1704-1708.

[30] G. Nordendorf, O. Kasdorf, H. S. Kitzerow, Y. Liang, X. Feng, K. Müllen, Jpn. J. Appl. Phys. 2010, 49, 100206.

[31] M. Wahle, O. Kasdorf, H. S. Kitzerow, Y. Liang, X. Feng, K. Müllen, Mol. Cryst. Liq. Cryst. 2011, 543, 187-193.

[32] A. C. Ferrari, J. C. Meyer, V. Scardaci, C. Casiraghi, M. Lazzeri, F. Mauri, S. Piscanec, D. Jiang, K. S. Novoselov, S. Roth, A. K. Geim, Phys. Rev. Lett. 2006, 97, 187401.

[33] J. Kang, S. Hwang, J. H. Kim, M. H. Kim, J. Ryu, S. J. Seo, B. H. Hong, M. K. Kim, J. B. Choi, ACS Nano 2012, 6, 5360-5365.

[34] P. Raynes, in Handbook of Visual Display Technology (Eds: J. L. Chen, W. Cranton, M. Fihn), Springer Verlag, Berlin/Heidelberg 2012, pp.1433-1444.

[35] D. Y. Wang, I. S. Huang, P. H. Ho, S. S. Li, Y. C. Yeh, D. W. Wang, W. L. Chen, Y. Y. Lee, Y. M. Chang, C. C. Chen, C. T. Liang, C. W. Chen, Adv. Mater. 2013, 25, 4521-4526.

[36] W. J. Choi, Y. J. Chung, S. Park, C. S. Yang, Y. K. Lee, K. S. An, Y. S. Lee, J. O. Lee, Adv. Mater. 2014, 26, 637-644.

[37] A. Kasry, M. A. Kuroda, G. J. Martyna, G. S. Tulevski, A. A. Bol, ACS Nano 2010, 4, 3839-3844.

[38] J. G. Ok, M. K. Kwak, C. M. Huard, H. S. Youn, L. J. Guo, Adv. Mater. 2013, 25, 6554-6561.

[39] J. Y. Chen, Y. L. Guo, Y. G. Wen, L. P. Huang, Y. Z. Xue, D. C. Geng, B. Wu, B. R. Luo, G. Yu, Y. Q. Liu, Adv. Mater. 2013, 25, 992-997. 